\title{
A Backward Monte Carlo Method for Simulation of the Electron Quantum Kinetics in Semiconductors
}

\author{
M. NEDJALKOV ${ }^{\mathrm{a}, *}$, H. KOSINA ${ }^{\mathrm{a}}, \mathrm{S}$ SELBERHERR ${ }^{\mathrm{a}}$ and I. DIMOV ${ }^{\mathrm{b}}$ \\ ${ }^{a}$ Institute for Microelectronics, TU-Vienna, Gusshausstrasse 27-29/E360, A-1040 Wien, Austria; \\ ${ }^{\mathrm{b}}$ CLPP, Bulgarian Academy of Sciences, Sofia, Bulgaria
}

\begin{abstract}
A quantum-kinetic equation accounting for the electron-phonon interaction is solved by a stochastic approach. Analyzed are three analytically equivalent integral formulation of the equation which appear to have different numerical properties. Particularly the path-integral formulation is found to be advantageous for the numerical treatment. The analysis is supported by the presented simulation results. A variety of physical effects such as collisional broadening and collision retardation introduced by the equation are discussed.
\end{abstract}

Keywords: Monte Carlo; Quantum transport; Femtosecond relaxation; Electron-phonon interaction; Collisional broadening; Memory effects; Integral equations

\section{INTRODUCTION}

We introduce an equation which describes the quantum kinetics of a semiconductor carrier system coupled with a phonon bath. The time evolution of such system is predestinated by the initial state. On a quantum-kinetic level the knowledge of the carrier-phonon initial state is often a problematic task. In this respect it is convenient to consider carries generated by a laser pulse at low temperatures, a case with no carriers at the beginning of the excitation. The relevant description of the phenomena is given by the semiconductor Bloch equations accounting for the carrier-phonon, carrier-photon and carrier-carrier interactions and interference effects [7]. In order to concentrate on the carrier-phonon kinetics only, a simplified consideration is needed, given by the one-band model [4]. It describes a relaxation of an initial distribution of carriers i.e., the phonon interaction is switched on after the laser pulse completed the carrier generation. Despite that a generation term is more realistic than the initial condition, the latter allows to concentrate on the quantum-kinetic aspects of the electron-phonon interaction. The one-band model is obtained in the framework of the density matrix formalism. The Hamiltonian $H=\Sigma_{\mathbf{k}} \varepsilon_{\mathbf{k}} c_{\mathbf{k}}^{+} c_{\mathbf{k}}+\Sigma_{\mathbf{q}} \omega b_{\mathbf{q}}^{+} b_{\mathbf{q}}+\Sigma_{\mathbf{k}^{\prime}, \mathbf{k}}$ $\left(g_{\mathbf{q}} c_{\mathbf{k}^{\prime}}^{+} b_{\mathbf{k}^{\prime}-\mathbf{k}} c_{\mathbf{k}}+c c\right)$ accounts for Froehlich interaction with coupling $g_{\mathbf{k}^{\prime}-\mathbf{k}}, c_{\mathbf{k}}^{+}\left(c_{\mathbf{k}}\right)$ and $b_{\mathbf{q}}^{+}\left(b_{\mathbf{q}}\right)$ are the electron and phonon creation(annihilation) operators respectively, $\varepsilon_{\mathbf{k}}=h^{2} k^{2} / 2 m$ is the electron

*Corresponding author. e-mail: nedjalkov@iue.tuwien.ac.at 
energy, and $\omega$ is the phonon frequency. The physical variables are statistical averages $\langle\cdot\rangle$ of combinations of creation and annihilation operators. Relevant are the electron and phonon distributions $f(\mathbf{k}, t)=\left\langle c_{\mathbf{k}}^{+} c_{\mathbf{k}}\right\rangle, n_{q}(\mathbf{q}, t)=\left\langle b_{\mathbf{q}}^{+} b_{\mathbf{q}}\right\rangle$, Their equations of motion introduce the phonon assisted density matrices $s\left(\mathbf{k}^{\prime}, \mathbf{k}, t\right)=(i / h) g_{\mathbf{k}^{\prime}-\mathbf{k}}\left\langle c_{\mathbf{k}^{\prime}}^{+} b_{\mathbf{k}^{\prime}-\mathbf{k}} c_{\mathbf{k}}\right\rangle$. The equations for $s$ introduce averages of four operators and so forth, leading to an infinite set (the BBGKY hierarchy) of equations. The set is closed by approximations in the equations of motion of the four operator averages. First the five operator terms are factorized into distribution functions and phonon assisted density matrices. Afterwards adiabatic and Markov approximations are performed and the result is used in the equations for $s$. The linearized one-band model, under the assumption of equilibrium phonons is given by the equations:

$$
\begin{aligned}
\frac{d}{d t} f(\mathbf{k}, t)= & 2 \sum_{\mathbf{k}^{\prime}} \operatorname{Re}\left[s\left(\mathbf{k}^{\prime}, \mathbf{k}, t\right)-s\left(\mathbf{k}, \mathbf{k}^{\prime}, t\right)\right] \\
\frac{d}{d t} s\left(\mathbf{k}^{\prime}, \mathbf{k}, t\right)= & \left(i \Omega\left(\mathbf{k}^{\prime}, \mathbf{k}\right)-\Gamma\left(\mathbf{k}^{\prime}, \mathbf{k}\right)\right) s\left(\mathbf{k}^{\prime}, \mathbf{k}, t\right) \\
& +\frac{1}{\hbar^{2}}\left\|g_{\mathbf{k}^{\prime}-\mathbf{k}}\right\|^{2} \\
& \times\left[f\left(\mathbf{k}^{\prime}, t\right)(n+1)-f(\mathbf{k}, t) n\right]
\end{aligned}
$$

which are supplemented by initial conditions $f(\mathbf{k}$, $0)=\phi(\mathbf{k}), s\left(\mathbf{k}^{\prime}, \mathbf{k}, 0\right)=0$.

Here $\Omega\left(\mathbf{k}^{\prime}, \mathbf{k}\right)=\left(\varepsilon\left(\mathbf{k}^{\prime}\right)-\varepsilon(\mathbf{k})-\hbar \omega\right) / \hbar, n$ is the Bose distribution, the damping $\Gamma\left(\mathbf{K}^{\prime}, \mathbf{k}\right)=\lambda(\mathbf{k})+$ $\lambda\left(\mathbf{k}^{\prime}\right)$ is related to the finite carrier lifetime against the scattering process: $\lambda(\mathbf{k})=\int d^{3} q\left(V / 2^{3} \pi^{2} \hbar\right)$ $\Sigma_{ \pm}\left\|g_{\mathbf{k}^{\prime}-\mathbf{k}}\right\|^{2} \delta\left(\varepsilon\left(\mathbf{k}^{\prime}\right)-\varepsilon(\mathbf{k}) \pm \hbar \omega\right) \quad(n+1 / 2 \pm 1 / 2)$. This equation set can be further processed [5] if (2) is integrated formally and inserted in (1) which leads to:

$$
\begin{aligned}
\frac{\partial}{\partial t^{\prime}} f\left(\mathbf{k}, t^{\prime}\right)=\int_{0}^{t^{\prime}} d t^{\prime \prime} \int d \mathbf{k}^{\prime} & \left\{S\left(\mathbf{k}^{\prime}, \mathbf{k}, t^{\prime}-t^{\prime \prime}\right)\right. \\
& \left.-S\left(\mathbf{k}, \mathbf{k}^{\prime}, t^{\prime}-t^{\prime \prime}\right) f\left(k, t^{\prime \prime}\right)\right\}
\end{aligned}
$$

$$
\begin{aligned}
& S\left(\mathbf{k}^{\prime}, \mathbf{k}, t^{\prime}-t^{\prime \prime}\right) \\
& =\frac{V}{(2 \pi)^{3} \hbar^{2}}\left\|g_{\mathbf{k}^{\prime}-\mathbf{k}}\right\|^{2} e^{-\left(\Gamma\left(\mathbf{k}^{\prime}, \mathbf{k}\right)\right)\left(t^{\prime}-t^{\prime \prime}\right)} \\
& \quad \times\left\{\cos \left(\Omega\left(\mathbf{k}^{\prime}, \mathbf{k}\right)\left(t^{\prime}-t^{\prime \prime}\right)\right)(n+1)\right. \\
& \left.\quad+\cos \left(\Omega\left(\mathbf{k}, \mathbf{k}^{\prime}\right)\left(t^{\prime}-t^{\prime \prime}\right)\right) n\right\}
\end{aligned}
$$

The result can be recognized as the zero electric field form of the quantum-kinetic equation reported in [3], which is now obtained by an alternative to the projection technique way.

It has been recognized that the numerical evaluation of (3) is a formidable task and that a relevant approach is the Monte Carlo (MC) method [3]. The numerical method used here is a formal extension of the Backward MC approach for semiclassical [1] and quantum transport [2] simulations. The method utilizes the theory of stochastic algorithms for solving integral equations. The convergence of iteration series of the concrete integral equation significantly affects the efficiency of the method. In the next section we introduce three different integral forms of (3). They allow to analyze a variety of physical and numerical aspects of the quantum-kinetic equation and the numerical method, presented in the last section.

\section{INTEGRAL FORMS}

The first integral form of (3) is obtained by a direct integration over $t^{\prime}$ in the limits $(0 ; t)$ and using the initial condition on the right hand side. The equation gives rise to a second integral form obtained after the following transformations. The order of the two time integrals can be exchanged according to $\int_{0}^{t} d t^{\prime} \int_{0}^{t \prime} d t^{\prime \prime}=\int_{0}^{t} d t^{\prime \prime} \int_{t^{\prime \prime}}^{t} d t^{\prime}$. Furthermore the kernel $S$ can be analytically integrated over $t^{\prime}$ with the help of the identity:

$$
\begin{aligned}
\int_{0}^{t-t^{\prime \prime}} & d \tau e^{-\left(\Gamma\left(\mathbf{k}^{\prime}, \mathbf{k}\right)\right) \tau} \cos \left(\Omega\left(\mathbf{k}^{\prime}, \mathbf{k}\right) \tau\right) \\
= & L\left(\mathbf{k}^{\prime}, \mathbf{k}\right)+L\left(\mathbf{k}^{\prime}, \mathbf{k}\right) \\
& \times\left(\frac { \Omega ( \mathbf { k } ^ { \prime } , \mathbf { k } ) } { \Gamma ( \mathbf { k } ^ { \prime } , \mathbf { k } ) } \operatorname { s i n } \left(\Omega\left(\mathbf{k}^{\prime}, \mathbf{k}\right)\left(t-t^{\prime \prime}\right)\right.\right. \\
& \left.\quad-\cos \left(\Omega\left(\mathbf{k}^{\prime}, \mathbf{k}\right)\left(t-t^{\prime \prime}\right)\right)\right) e^{-\left(\Gamma\left(\mathbf{k}^{\prime}, \mathbf{k}\right)\right)\left(t-t^{\prime \prime}\right)}
\end{aligned}
$$


where $L$ is a Lorentzian function $L\left(\mathbf{k}^{\prime}, \mathbf{k}\right)=$ $\left(\Gamma\left(\mathbf{k}^{\prime}, \mathbf{k}\right) / \Omega^{2}\left(\mathbf{k}^{\prime}, \mathbf{k}\right)+\Gamma^{2}\left(\mathbf{k}^{\prime}, \mathbf{k}\right)\right)$. Thus the scattering term denoted by $\mathcal{L}\left(\Gamma\left(\mathbf{k}^{\prime}, \mathbf{k}\right) ; \mathbf{k}^{\prime}, \mathbf{k}, t-t^{\prime \prime}\right)=$ $\int_{t^{\prime \prime}}^{t} d t^{\prime} S\left(\mathbf{k}^{\prime}, \mathbf{k}, t^{\prime}-t^{\prime \prime}\right)$ is decomposed into a time independent part (two Lorentzian multiplied by the equilibrium phonon factors) and an oscillating, exponentially damped function of the evolution time. The two parts cancel each other at $t=0$. The Markovian limit $t \rightarrow \infty$ of $\mathcal{L}$ is presented by the Lorentzian part. The time dependent part is liable for the memory character of the equation.

$$
\begin{aligned}
f(\mathbf{k}, t)=\int_{0}^{t} d t^{\prime \prime} & \int d \mathbf{k}^{\prime}\left(\mathcal{L}\left(\Gamma\left(\mathbf{k}^{\prime}, \mathbf{k}\right) ; \mathbf{k}^{\prime}, \mathbf{k}, t-t^{\prime \prime}\right)\right. \\
& \times f\left(\mathbf{k}^{\prime}, t^{\prime \prime}\right)-\mathcal{L}\left(\Gamma\left(\mathbf{k}^{\prime}, \mathbf{k}\right) ; \mathbf{k}, \mathbf{k}^{\prime}, t-t^{\prime \prime}\right) \\
& \left.\times f\left(\mathbf{k}, t^{\prime \prime}\right)\right)+\phi(k)
\end{aligned}
$$

The Markovian limit of this equation does not recover the semiclassical Boltzmann equation. It is due to the finite lifetime of the carriers - the energy conserving delta function is recovered by the limit $\Gamma \rightarrow 0$.

The derivation of the third integral form utilizes the main idea of the path integral transformation, which is the basis of the MC calculations in the Boltzmann transport framework. A term $\psi(\mathbf{k}) f(\mathbf{k}$, $\left.t^{\prime}\right)$, where $\psi$ is a positive function is added to both sides of (3). The left hand side can be written as $e^{-\psi(\mathbf{k}) t^{\prime}}\left(d / d t^{\prime}\right)\left(e^{\psi(\mathbf{k}) t^{\prime}} f\left(\mathbf{k}, t^{\prime}\right)\right)$. The equation is further divided by $e^{-\psi(\mathbf{k}) t^{\prime}}$ and integrated on $t^{\prime}$ in the interval $(0 ; t)$. A subsequent division on $e^{\psi(\mathbf{k}) t}$ leads to path integral formulation, with $f(\mathbf{k}, t)$ on the left and the exponential damping due to the $\psi$ function incorporated in the time integrals on the right. The identity (4) still can be applied to give:

$$
\begin{array}{r}
f(\mathbf{k}, t)=\int_{0}^{t} d t^{\prime \prime} \int d \mathbf{k}^{\prime} e^{-\psi(\mathbf{k})\left(t-t^{\prime \prime}\right)} \mathcal{L}\left(\Gamma\left(\mathbf{k}^{\prime}, \mathbf{k}\right)\right. \\
\left.-\psi(\mathbf{k}) ; \mathbf{k}^{\prime}, \mathbf{k}, t-t^{\prime \prime}\right) f\left(\mathbf{k}^{\prime}, t^{\prime \prime}\right) \\
-\int_{0}^{t} d t^{\prime \prime} e^{-\psi(\mathbf{k})\left(t-t^{\prime \prime}\right)} \\
\times\left(\int d \mathbf { k } ^ { \prime } \mathcal { L } \left(\Gamma\left(\mathbf{k}^{\prime}, \mathbf{k}\right)-\psi(\mathbf{k}) ;\right.\right. \\
\left.\left.\mathbf{k}, \mathbf{k}^{\prime}, t-t^{\prime \prime}\right)-\psi(\mathbf{k})\right) \\
\times f\left(\mathbf{k}, t^{\prime \prime}\right)+\phi(k) e^{-\psi(\mathbf{k}) t}
\end{array}
$$

This path integral form coincides with the zero field Barker-Ferry equation [3] with the only modification, that the self-scattering constant is replaced by the function $\psi$. The advantages of the Barker-Ferry form for the utilized numerical approach are analyzed in the next section. The physical aspects of the quantum model and particularly of its Lorentzian limit are discussed and demonstrated by simulation experiments.

\section{RESULTS AND DISCUSSIONS}

The simulation results are obtained for GaAs with material parameters taken from [4]. The initial condition is given by a Gaussian distribution in energy, corresponding to a 87 femtosecond laser pulse with an excess energy of $180 \mathrm{meV}$, scaled in a way to ensure peak value equal to unity. Zero lattice temperature has been chosen in order to allow a convenient comparison with the behavior of semiclassical electrons. At such temperature the latter can only emit phonons and loose energy equal to a multiple of the phonon energy $\hbar \omega$. The evolution of the distribution function is patterned by replicas of the initial distribution shifted towards low energies. The electrons can not be scattered out of the states below the phonon energy and can not appear above the initial distribution. This simple semiclassical behavior will be the reference background for the effects imposed by the quantum-kinetic Eq. (3). The symmetry of the task allows to use spherical coordinates with a wave vector amplitude $k=|\mathbf{k}|$. The figures present the quantity $k f(k, t)$, the distribution function multiplied by the density of states, in arbitrary units versus $k^{2}\left(10^{14} / \mathrm{m}^{2}\right)$, which is proportional to the electron energy.

\subsection{Physical Aspects}

Figure 1 shows quantum solutions for low evolution times. Electrons appear in the semiclasically forbidden region above the initial condition. This is explained by referring to the scattering term $S$ in the first integral form. For small time differences in 


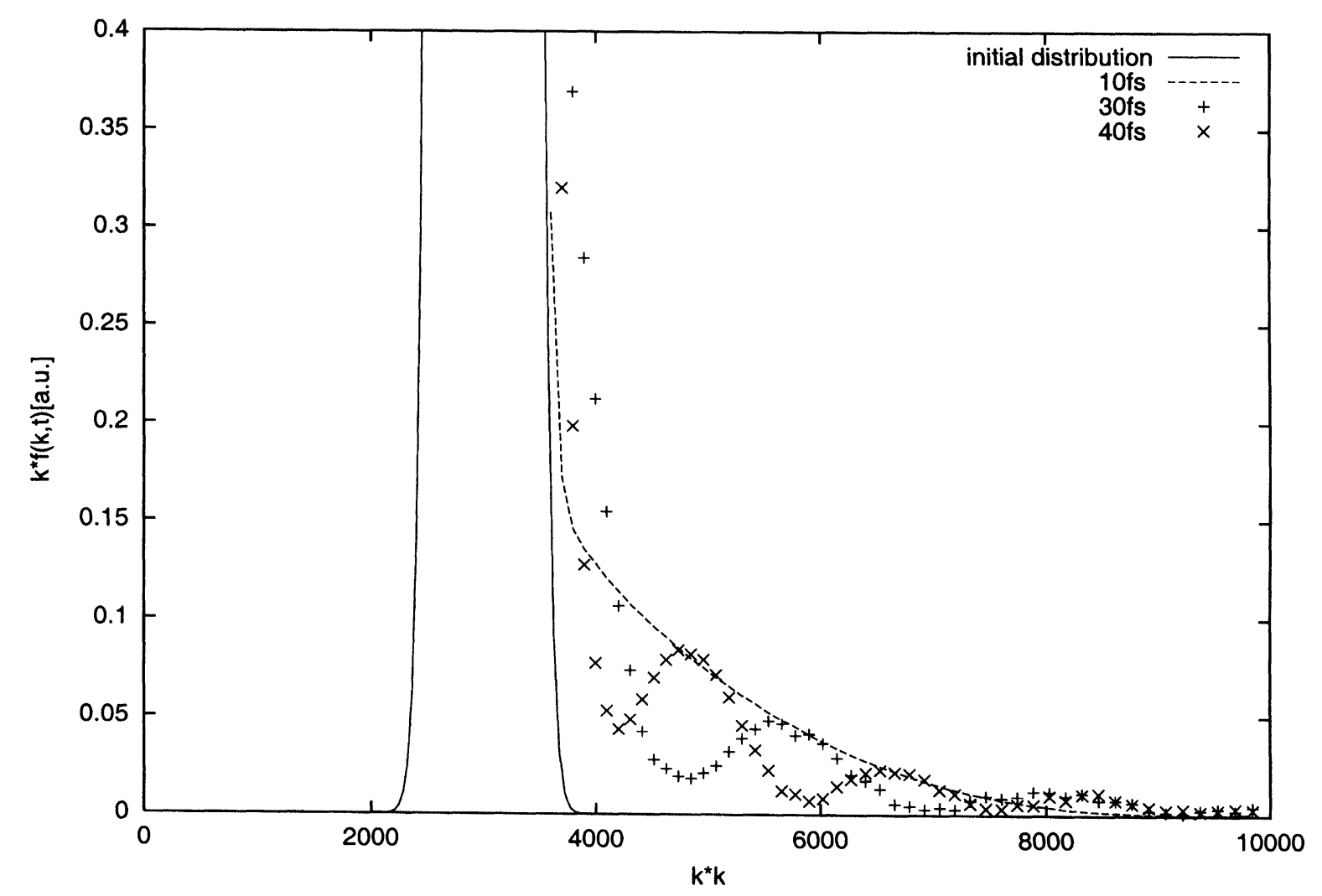

FIGURE 1 Quantum solutions for three low evolution times. Electrons appear in the semiclasically forbidden region above the initial condition.

the cosine function the probability for scattering into the whole Brillouin zone becomes finite. Despite that only a small fraction of the electrons populate the higher energy states - the resolution is within four orders of magnitude below the initial peak value - this property remains even if a generation term is considered. The initial condition allows a clear demonstration of the effect.

The quantum effects in the energy region below the initial condition can be interpreted with the help of the scattering term $\mathcal{L}$ of the second integral form. Itself the time independent part of $\mathcal{L}$ is responsible for the effect of collisional broadening, destroying the replica-like pattern of the distribution function. Figure 2 compares the semiclassical distribution after $400 \mathrm{fs}$ with the solution of a Boltzmann like equation (BLE), where the delta function in energy is replaced by the Lorentzian. A detailed discussion of the effects delivered by the Lorentzian model are given in [6].

The memory character of the equation, carried on by the time-dependent part of $\mathcal{L}$, introduces a collision retardation. The latter is demonstrated in Figures 3 and 4 as an delay in the build up of the remote peaks of the quantum solutions as compared to the corresponding BLE solutions.

At high evolution times the time independent part dominates the kinetics and introduces additional deviations from the semiclassical behavior. Due to the long reaching tails of the Lorentzian function, the electrons with energy below the LO phonon threshold are in mutual exchange, having an out-scattering rate of order of $10^{-5} / f s$. Furthermore a fraction of electrons run away 


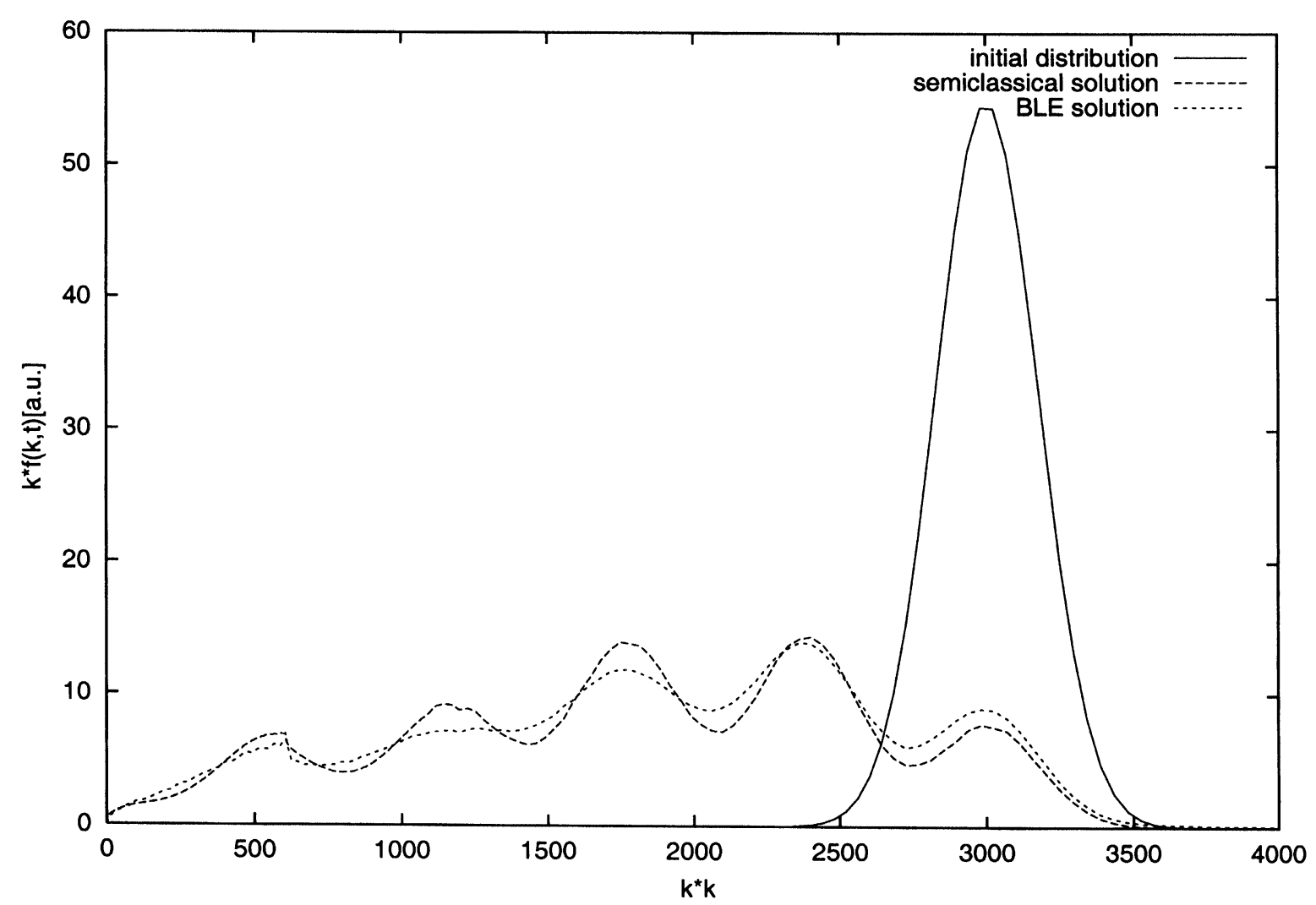

FIGURE 2 Semiclassical and BLE solutions for $400 \mathrm{fs}$ evolution time. The Lorentzian destroys the peak-like pattern in the region of low energies.

towards the high energy states leading to an artificial heating of the electron system [6]. Thus the application of (3) for high evolution times must be handled with care.

\subsection{Numerical Aspects}

The applied Monte Carlo method is based on the following estimator:

$$
\nu_{i}\left(x_{0}\right)=\frac{\mathcal{K}\left(x_{0}\right), x_{1}}{P\left(x_{0}, x_{1}\right)} \ldots \frac{\mathcal{K}\left(x_{i-1}, x_{i}\right)}{P\left(x_{i-1}, x_{i}\right)} \phi\left(x_{i}\right)
$$

which calculates the multiple integrals forming the iteration series of the integral equation: $f(x)=\int d x^{\prime} \mathcal{K}\left(x, x^{\prime}\right) f\left(x^{\prime}\right)+\phi(x)$, [5]. Here $x_{0}$ is the desired point $k, t$ where the solution is to be evaluated and $x_{i}, i>0$ given by the set of the integral variables: $k^{\prime}, t^{\prime}, t^{\prime \prime}$ for the first form and $k^{\prime}$, $t^{\prime \prime}$ for the second and third form and the BLE. An even transition probability density $P$ has been chosen for the all variables in the first form. For the rest of the equations $k$ has a Lorentzian distribution in the phase space, and the time is generated according the exponential distribution. The advantages of the method lie in the direct evaluation of the functional value at the desired point - in contrast the Ensemble MC provides only averaged estimates. A direct control of the numerical precision in the desired point is available. This is demonstrated by the high resolution of the statistical results on Figure 1. The method does not require the knowledge of the distribution function dependence on $k$ at previous times. 


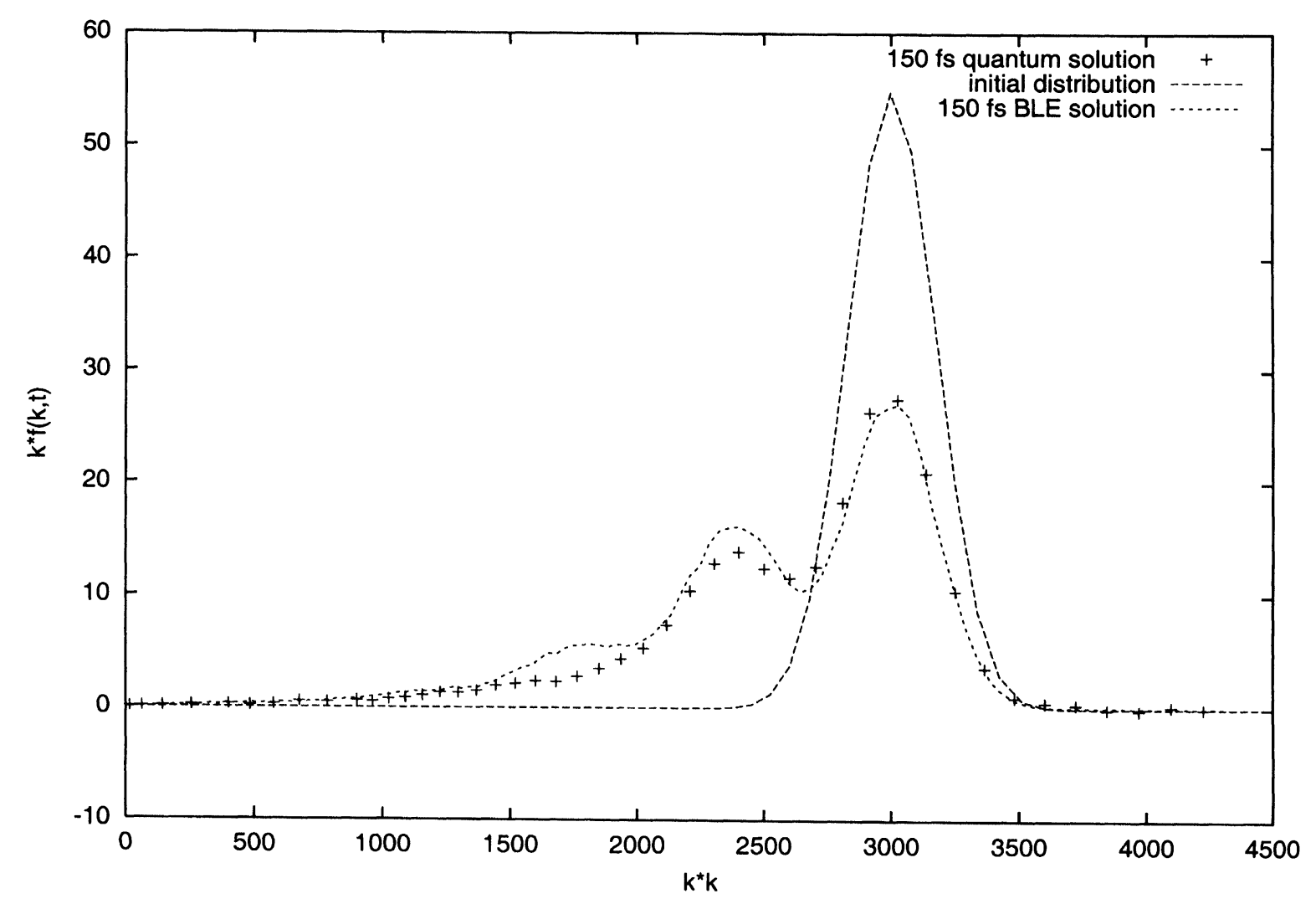

FIGURE 3 Quantum and BLE solutions for $150 \mathrm{fs}$ evolution time. The quantum solution follows the BLE solution with a delay.

The variance of the estimator for the first integral form rapidly increases, allowing simulations up to $100 \mathrm{fs}$ evolution time. The mean value of the estimator is formed by cancellations of positive and negative numbers, which rapidly increase with the evolution time. The analytic evaluation of one time integral leading to the second integral form only slightly improves the variance. This shows that the reason is in the poor convergence of the iteration series rather than in the stochastic error in the evaluation of the integrals. The build up of the Markovian limit of (4) increases the number of the relevant iteration terms before the truncation of the series. The same problem has been reported for the direct time integral form of the Boltzmann equation [2]. The remedy in the semiclassical case is in the path integral formulation of the equation. It evaluates analytically the negative contributions of the out-scattering term by the exponential function, giving the probability for the free-flight time. The same effect is observed in the path integral form (6). The function $\psi$ subtracted from the second term gives rise to an exponential damping with the evolution time, which improves the convergence of the iteration series. The above analysis is supported by the numerical experiments. A choice of $\psi=\lambda$ has been done, which simplifies the scattering term. Despite that the Lorentzian in (6) have higher peak values as compared to (4), evolution times of $300 \mathrm{fs}$ can be conveniently simulated. For the BLE $\psi$ has been chosen to cancel exactly the out-scattering term. 


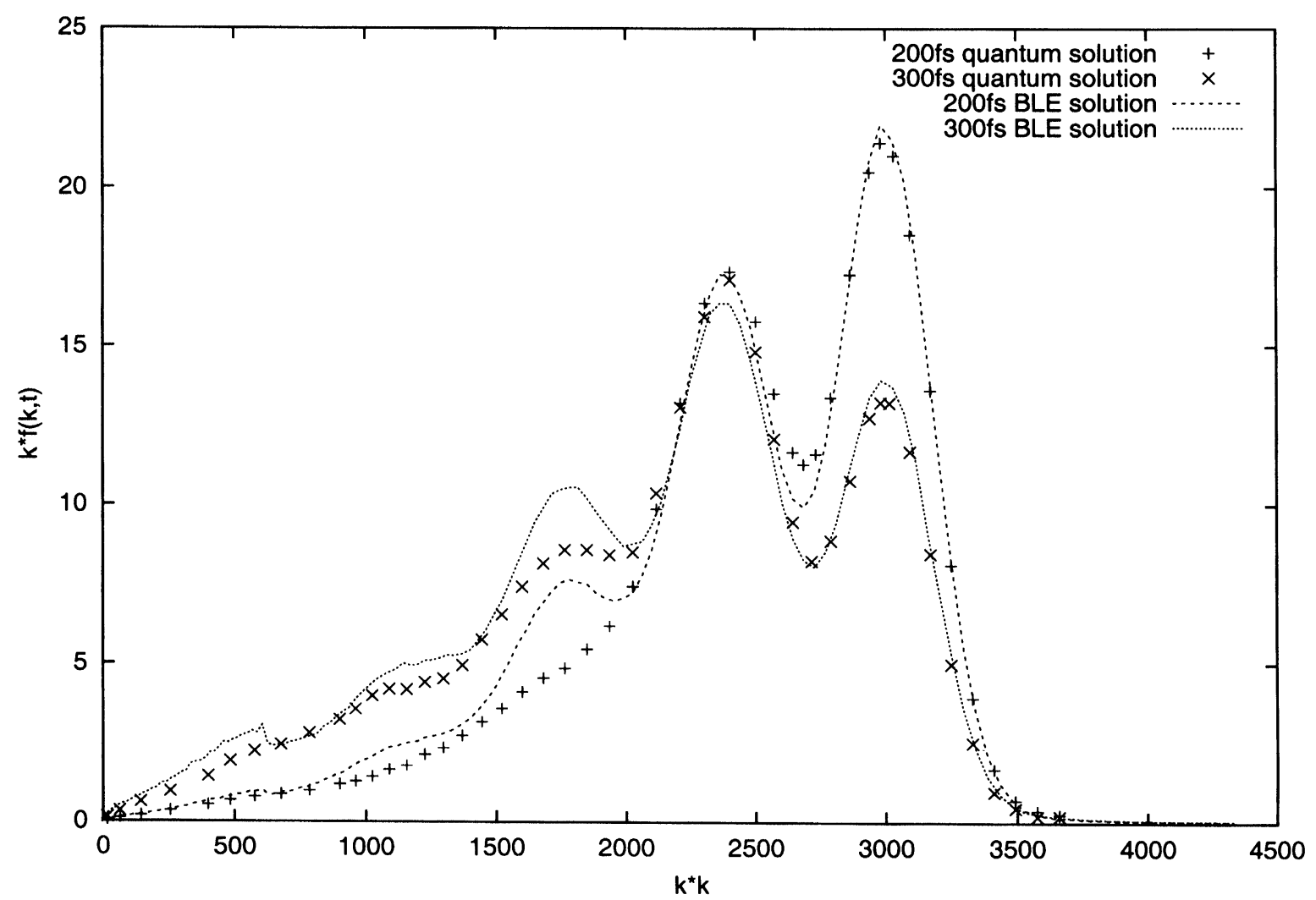

FIGURE 4 Quantum and BLE solutions for $200 \mathrm{fs}$ and $300 \mathrm{fs}$ evolution time.

\section{Acknowledgment}

This work has been partly supported by the European Commission Project NANOTCAD, IST-1999-10828.

\section{References}

[1] Brunetti, R., Jacoboni, C. and Rossi, F., Quantum theory of transient transport in semiconductors: A Monte Carlo approach. Physical Review B, 39(15), 10781-10790, May, 1989.

[2] Jacoboni, C., Poli, P. and Rota, L. (1988). A new Monte Carlo technique for the solution of the Boltzmann transport equation. Solid-State Electron., 31(3/4), $5523-526$.
[3] Barker, J. and Ferry, D. (1979). Self-scattering pathvariable formulation of high field, time-dependent quantum kinetic equations for semiconductor transport in the finitecollision-duration regime. Physical Review Letters, 42(26), $1779-1781$.

[4] Schilp, J., Kuhn, T. and Mahler, G. (1994). Electronphonon quantum kinetics in pulse-excited semiconductors: Memory and renormalization effects. Physical Review B, 50(8), $5435-5447$.

[5] Nedjalkov, M. and Dimov, I. (1998). Statistical modelling of pulse excited electron quantum kinetics in one band semiconductor. Mathematics and Computers in Simulations, 47.

[6] Nedjalkov, M., Dimov, I. and Haug, H. (1998). Numerical studies of the markovian limit of the quantum kinetics with phonon scattering. Phys. stat. sol. (b), 209, 109-121.

[7] Kuhn, T. and Rossi, F. (1992). Monte Carlo simulation of ultrafast processes in photoexcited semiconductors: Coherent and incoherent dynamics. Physical Review B, 46(12), 7496-7514. 

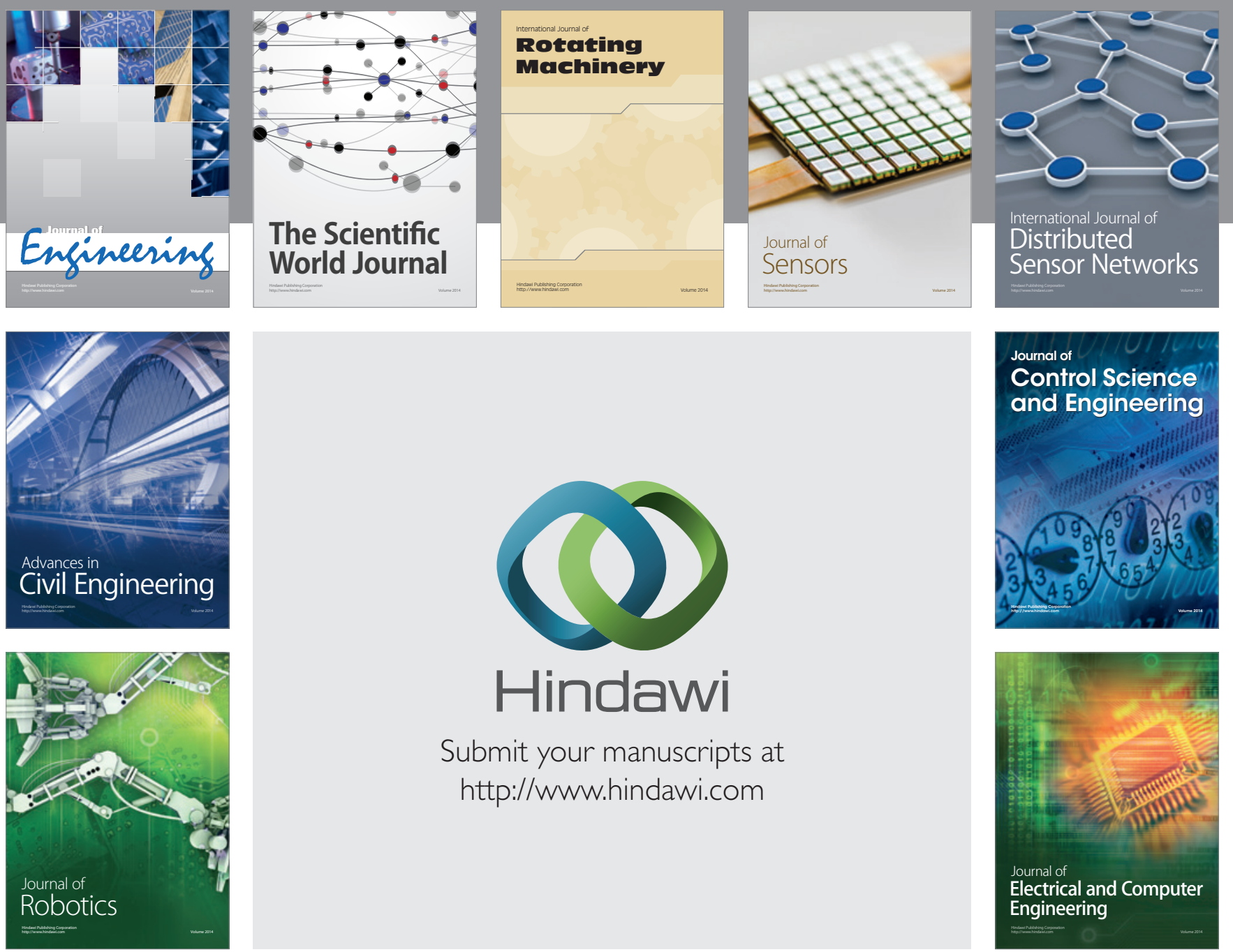

Submit your manuscripts at

http://www.hindawi.com
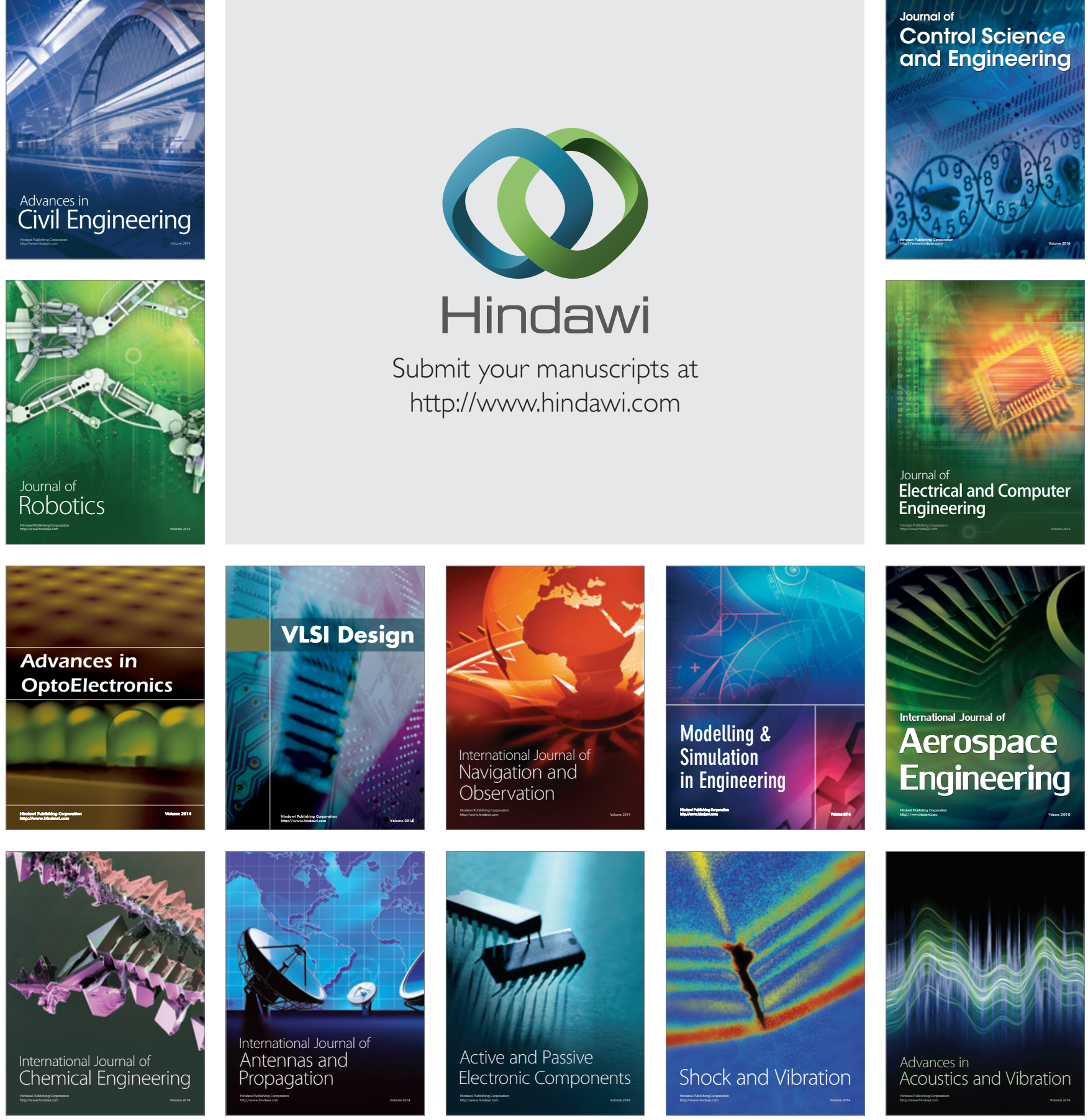